ROCKY MOUNTAIN

JOURNAL OF MATHEMATICS

Volume 36, Number 6, 2006

\title{
ON THE COMPACTNESS OF THE SET OF BOUNDED ORBITS FOR A DIFFERENTIAL SYSTEM
}

\author{
YU SHU-XIANG \\ Dedicated to Professor Ye Yan-Qian on his 80th birthday.
}

ABSTRACT. In this paper, an answer in a special case to a problem proposed by Conley is given.

1. Introduction. In $[2]$, Conley studied the flows on $R^{n}$ such that the set of bounded orbits is compact. In particular, he proposed the following problem, see $[\mathbf{2}$, p. 31]:

Problem. Suppose $V(x)$ is a smooth function on $R^{n}$ with $\|\nabla V\| \geq \varepsilon$ for $|x| \geq R$. Is it true that the set of bounded solutions of $d x / d t=y$, $d y / d t=\theta y-\nabla V(x)$ is compact whenever $\theta \neq 0$ ?

In the present paper, we shall give a positive answer in the case $n=1$.

2. The main result. When $n=1$, the differential system in the problem is the following planar system

$$
\begin{aligned}
& \frac{d x}{d t}=y, \\
& \frac{d y}{d t}=\theta y-\frac{d V}{d x},
\end{aligned}
$$

where $\theta \neq 0$, without loss of generality we assume that $\theta>0$ (when $\theta<0$ one can make a change $t \rightarrow-t, y \rightarrow-y$ ); and $V(x)$ is a smooth scalar function such that following condition is satisfied:

$$
\left|\frac{d V}{d x}\right| \geq \varepsilon \quad \text { when } \quad|x| \geq R .
$$

It is easy to show the following properties of the system (1).

2000 AMS Mathematics Subject Classification. Primary 34C.

Research supported by the National Science Foundation of China.

Received by the editors on January 14, 2004.

Copyright (c)2006 Rocky Mountain Mathematics Consortium 
(i) By [2, pp. 12-13], a system is called gradient-like if there is some continuous real valued function which is strictly decreasing on nonconstant solutions. It is easy to see that the system (1) is gradientlike with respect to the function

$$
g(x, y)=-\left(\frac{y^{2}}{2}+V(x)\right)
$$

In fact, the derivative of $g$ on solutions is as follows:

$$
\left.\frac{d g}{d t}\right|_{(1)}=-\theta y^{2}
$$

Therefore $g$ is at least nonincreasing on solutions. Further, from the first expression of (1) it follows that there are not any nonconstant solutions lying on the straight line $y=0$ over some time interval. Hence, $g$ is strictly decreasing on nonconstant solutions. This means that the system (1) is gradient-like.

(ii) From the above definition of a gradient-like system, it follows that the system (1) has no closed orbits. Moreover, the $\omega$-limit set (or the $\alpha$-limit set) of any bounded orbit of the system (1) consists of critical points. Any nonconstant bounded orbit of the system (1) must tend to distinct critical points as $t \rightarrow+\infty$ and as $t \rightarrow-\infty$, respectively.

(iii) Let $W$ denote the union of all bounded orbits of the system (1). Clearly, each critical point of the system (1) can be denoted by $\left(x_{0}, 0\right)$, where $x_{0}$ satisfies $d V / d x\left(x_{0}\right)=0$. Let $D$ denote the set of all critical points of (1). If $d V / d x \neq 0$ for all $x$, then $D$ is empty. Hence, from the property (ii) it follows that $W$ is empty. If $d V / d x$ has only one zero $x_{1}$, then $W$ consists of the critical point $\left(x_{1}, 0\right)$. Therefore, in what follows, it will suffice to consider the case that $d V / d x$ has at least two zeros. Since the set of zeros of $d V / d x$ is closed and the condition (2) implies that it is also bounded, it follows that this set is compact. Thus, there must be a maximal value $x=b$ and a minimal value $x=a$ such that all zeros of $d V / d x$ lie entirely in the closed interval $[a, b]$ of the $x$-axis. 
Then we distinguish four cases:

$$
\begin{aligned}
& \text { (I) } \frac{d V}{d x}>0 \text { for } x>b \text { and } \frac{d V}{d x}<0 \text { for } x<a \\
& \text { (II) } \frac{d V}{d x}<0 \text { for } x>b \text { and } \frac{d V}{d x}>0 \text { for } x<a \\
& \text { (III) } \frac{d V}{d x}>0 \text { for } x>b \text { and } \frac{d V}{d x}>0 \text { for } x<a \\
& \text { (IV) } \frac{d V}{d x}<0 \text { for } x>b \text { and } \frac{d V}{d x}<0 \text { for } x<a \text {. }
\end{aligned}
$$

In this paper, we shall prove the following theorem.

Theorem 1. If the set of bounded orbits of the system (1) is nonempty, then it must be compact.

3. Proof of the theorem. We now first introduce a result from [1], see [1, p. 910].

Consider the differential system

$$
\begin{aligned}
& \frac{d x}{d t}=X(x, y), \\
& \frac{d y}{d t}=Y(x, y),
\end{aligned}
$$

in the plane $R^{2}$ where $X$ and $Y$ are continuous and assume that solutions of arbitrary initial value problems are unique. Let the vector field $V=(X, Y)$ define a flow $f(p, t)$.

Let $B \subset R^{2}$ be the closure of a bounded and connected open set with the boundary $\partial B$. Let $L_{1} \ldots L_{n}$ denote its boundary components, where $L_{i} \cap L_{j}=\varnothing$ for $i \neq j$, and $L_{1}$ the external boundary. Each of them is a smooth simple closed curve. We define three subsets $b^{+}, b^{-}$, $\tau$ as follows:

$$
\begin{aligned}
b^{+} & =\{p \in \partial B \mid \exists \varepsilon>0 \text { with } f(p,(-\varepsilon, 0)) \cap B=\varnothing\}, \\
b^{-} & =\{p \in \partial B \mid \exists \varepsilon>0 \text { with } f(p,(0, \varepsilon)) \cap B=\varnothing\}, \\
\tau & =\{p \in \partial B \mid V \text { is tangent to } \partial B \text { at } p\} .
\end{aligned}
$$


Definition 1 [3]. If $b^{+} \cap b^{-}=\tau$ and $b^{+} \cup b^{-}=\partial B$, then $B$ is called an isolating block for the flow defined by (6).

The following is a theorem in [1].

Theorem 2. The set of bounded orbits of the system (6) is compact if and only if there is an isolating block $B$ of the system (6) such that all critical points and closed orbits of (6) are contained in the region $G$ enclosed by the external boundary $L_{1}$ of $B$.

Proof of Theorem 2. See [1, Theorem 2.1].

Proof of Theorem 1. As stated above, it will be enough to consider the case that $d V / d x$ has at least two zeros. Consider first case (I). From $d V / d x>0$ for $x>b$ and the condition (2), it follows that $d V / d x \geq \varepsilon$ for $x>R>b$. Hence, we have

$$
V(x)-V(b)=\int_{b}^{x} \frac{d V}{d x} d x=\int_{b}^{R} \frac{d V}{d x} d x+\int_{R}^{x} \frac{d V}{d x} d x>\varepsilon(x-R) .
$$

This implies that $V(x) \rightarrow+\infty$ as $x \rightarrow+\infty$. From $d V / d x<0$ for $x<a$ and the condition (2), it follows that $d V / d x \leq-\varepsilon$ for $x<-R<a$. Hence we have

$$
V(a)-V(x)=\int_{x}^{a} \frac{d V}{d x} d x=\int_{x}^{-R} \frac{d V}{d x} d x+\int_{-R}^{a} \frac{d V}{d x} d x<\varepsilon(x+R) .
$$

This implies that $V(x) \rightarrow+\infty$ as $x \rightarrow-\infty$. Let $F=-g=$ $\left(y^{2} / 2\right)+V(x)$. From (4), we have

$$
\left.\frac{d F}{d t}\right|_{(1)}=\theta y^{2} .
$$

Consider the family of curves

$$
F=\frac{y^{2}}{2}+V(x)-d=0,
$$

where $d$ is an arbitrary constant. Noting that $V(x) \rightarrow+\infty$ as $x \rightarrow \pm \infty$ together with the monotoneity of $V(x)$ as $x>b$ and as $x<a$, it is not 
difficult to check that there is a sufficiently large value $d_{0}>0$ such that the equation $V(x)-2 d=0$ determines a unique positive real number $x_{1}(d)>b$ and a unique negative real number $x_{2}(d)<a$ for each $d \geq d_{0}$. Hence, (8) defines a family of closed curves in the plane for all $d \geq d_{0}$. Each of these closed curves contains all critical points of the system (1) in its interior. As stated above, (7) means that $F$ is strictly increasing on nonconstant orbits. Take $d_{1}>d_{0}$, and let $T$ denote the interior region enclosed by the closed curve $F=d_{1}$. It is easy to see that the closure $\bar{T}$ is an isolating block. Obviously, all critical points of (1) are contained in $T$. Since the system (1) has no closed orbits, Theorem 2 implies that the set of bounded orbits of (1) is compact.

Secondly consider case (II). Let $\beta_{1}=\max _{a-1 \leq x \leq b+1}((1 / \theta) \cdot(d V / d x))$ and $\beta_{2}=\min _{a-1 \leq x \leq b+1}((1 / \theta) \cdot(d V / d x))$. Choose $M=(b+1,0)$ and construct the straight line $x=b+1$ through $M$. It intersects the straight line $y=\beta_{1}+1$ at $Z_{1}=\left(b+1, \beta_{1}+1\right)$ and the straight line $y=\beta_{2}-1$ at $Z_{2}=\left(b+1, \beta_{2}-1\right)$, respectively. Choose $N=(a-1,0)$ and construct the straight line $x=a-1$ through $N$. It intersects the straight line $y=\beta_{1}+1$ at $E_{1}=\left(a-1, \beta_{1}+1\right)$ and the straight line $y=\beta_{2}-1$ at $E_{2}=\left(a-1, \beta_{2}-1\right)$, respectively. Let $T_{1}$ denote the interior region enclosed by the curvilinear figure $M Z_{1} E_{1} N E_{2} Z_{2} M$ made up of the straight line segments $Z_{1} E_{1}, E_{1} E_{2}, E_{2} Z_{2}$ and $Z_{2} Z_{1}$. Let $\overline{T_{1}}$ denote its closure. From the second expression of (1) it follows that $d y / d t>0$ for all points on the line segment $E_{1} Z_{1}$ and $d y / d t<0$ on the line segment $E_{2} Z_{2}$. From the first expression (1) it follows that $d x / d t>0$ for all points on the half-open line segment $Z_{1} M$ and $E_{1} N$, and $d x / d t<0$ on the half-open line segment $Z_{2} M$ and $E_{2} N$. Since $d y / d t>0$ and $d x / d t=0$ at $M, M$ is an external tangency to $\overline{T_{1}}$. Similarly, $N$ is also an external tangency to $\overline{T_{1}}$. Clearly, no boundary point of $\overline{T_{1}}$ is on a solution curve which is contained completely in $\overline{T_{1}}$. Therefore, $\overline{T_{1}}$ is an isolating neighborhood of (1), see [2, pp. 3-4]. Let $S$ denote the maximal invariant set of (1) in $\overline{T_{1}}$. Clearly, all critical points of (1) are contained in $S$. By [3, p. 53] we know that for the isolated invariant set $S$ and the isolating neighborhood $\overline{T_{1}}$, one can construct an isolating block $B$ for $S$ which lies in $\overline{T_{1}}$. Then all critical points of (1) lies in $B$. Since the system (1) has no closed orbits, Theorem 2 implies that the set of bounded orbits of (1) is compact. 
Thirdly consider case (III). As stated in case (I), we have $d V / d x \geq \varepsilon$ for $x>R>b$ and $V(x) \rightarrow+\infty$ as $x \rightarrow+\infty$. Noting that $V(x) \rightarrow+\infty$ as $x \rightarrow+\infty$ together with the monotoneity of $V(x)$ as $x>b$, it is not difficult to check that there is a sufficiently large value $d_{2}>0$ such that the curve

$$
F_{1}=\frac{y^{2}}{2}+V(x)-d_{2}=0 \quad \text { and } \quad \mathrm{x} \geq b+1
$$

intersects the half axis $x>b+1$ at $M^{\prime}$ and the straight line $x=b+1$ at $Z_{1}^{\prime}=\left(b+1, \delta_{1}\right)$ and $Z_{2}^{\prime}=\left(b+1, \delta_{2}\right)$, respectively, where $\delta_{1}>\beta_{1}$ and $\delta_{2}<\beta_{2}$. Construct the straight line $y=\delta_{1}$ through $Z_{1}^{\prime}$. It intersects the straight line $x=a-1$ at $E_{1}^{\prime}$. Construct the straight line $y=\delta_{2}$ through $Z_{2}^{\prime}$. It intersects the straight line $x=a-1$ at $E_{2}^{\prime}$. Thus we obtain a closed curve $Z_{1}^{\prime} E_{1}^{\prime} E_{2}^{\prime} Z_{2}^{\prime} M^{\prime} Z_{1}^{\prime}$ made up of the curve (9) $Z_{2}^{\prime} M^{\prime} Z_{1}^{\prime}$ and the straight line segments $Z_{1}^{\prime} E_{1}^{\prime}, E_{1}^{\prime} E_{2}^{\prime}$, and $E_{2}^{\prime} Z_{2}^{\prime}$. Let $T_{2}$ denote the interior region of this closed curve and $\overline{T_{2}}$ its closure. Using the same argument used in cases (I) and (II), it follows that no boundary point of $\overline{T_{2}}$ is on a solution curve which is contained completely in $\overline{T_{2}}$. Therefore, $\overline{T_{2}}$ is an isolating neighborhood of (1). The same argument used in case (II) implies that the set of bounded orbits of (1) is compact.

Finally, consider case (IV). As stated in case (I), we have $V(x) \rightarrow+\infty$ as $x \rightarrow-\infty$. This fact together with the monotoneity of $V(x)$ as $x<a$ implies that there is a sufficiently large value $d_{3}>0$ such that the curve

$$
F_{2}=\frac{y^{2}}{2}+V(x)-d_{3}=0 \quad \text { and } \quad \mathrm{x} \leq a-1
$$

intersects the half axis $x<a-1$ at $N^{\prime}$ and the straight line $x=a-1$ at $E_{3}=\left(a-1, \delta_{3}\right)$ and $E_{4}=\left(a-1, \delta_{4}\right)$, respectively, where $\delta_{3}>\beta_{1}$ and $\delta_{4}<\beta_{2}$. Construct the straight line $y=\delta_{3}$ through $E_{3}$. It intersects the straight line $x=b+1$ at $Z_{3}$. Construct the straight line $y=\delta_{4}$ through $E_{4}$. It intersects the straight line $x=b+1$ at $Z_{4}$. Thus we obtain a closed curve $E_{3} N^{\prime} E_{4} Z_{4} Z_{3} E_{3}$ made up of the curve (10) $E_{3} N^{\prime} E_{4}$ and the straight line segments $E_{4} Z_{4}, Z_{4} Z_{3}$, and $Z_{3} E_{3}$. Let $T_{3}$ denote the interior region of this closed curve and $\overline{T_{3}}$ its closure. Then, the same argument used in case (III) implies that the set of bounded orbits of (1) is compact. This completes the proof of Theorem 1.

The following is a remark on Theorem 2 . 
Remark 1. Consider now the following system [2, p. 31]

$$
\begin{aligned}
& \frac{d x}{d t}=\frac{1-x^{2}}{\left(1+y^{2}\right)}, \\
& \frac{d y}{d t}=\frac{-2 x y\left(1-x^{2} / 3\right)}{\left(1+y^{2}\right)^{2}} .
\end{aligned}
$$

It is easy to see that this system has only two critical points $P_{1}=$ $(-1,0)$ and $P_{2}=(1,0)$. The lines $x= \pm 1$ and $y=0$ are invariant. From (11) we know that $d x / d t>0$ and $d y / d t$ has the sign of $-x y$ for all points of band region $-1<x<1$. This implies that the orbits with initial values $\left(0, y_{0}\right)$ tend to $P_{1}$ and $P_{2}$ respectively as $t \rightarrow-\infty$ and as $t \rightarrow+\infty$. Thus the set of bounded orbits is not compact, see [2].

It is not difficult to prove that there is no isolating block satisfying the conditions of Theorem 2. In fact, suppose that there is an isolating block $B$ such that the critical points $P_{1}$ and $P_{2}$ are contained in the region $G$ enclosed by the external boundary $L_{1}$ of $B$ (note that the system (11) has no closed orbits). Then, there is a sufficiently large value $y_{0}>0$ such that for increasing time the bounded orbit $\Gamma$ through $\left(0, y_{0}\right)$ leaves $G$ from $U_{1}$ (a strict exit point on $L_{1}$ ) and then enters $G$ at $U_{2}$ on $L_{1}$ so that the region $K$ enclosed by the orbit arc $U_{1} U_{2}$ and the segmental arc $U_{2} U_{1}$ of $L_{1}$ (corresponding to $y>0$ ) is a simply connected region. Lemma 3.1 (or Remark 3.1) in [1] implied that there must be at least one critical point of (11) in $K$. This is a contradiction.

\section{REFERENCES}

1. Ding Changming and $\mathrm{Yu}$ Shu-Xiang, A criterion on compactness of the set of bounded orbits, Nonlinear Anal. 46 (2001), 909-914.

2. C. Conley, Isolated invariant sets and the Morse index, Conf. Board Math. Sci. 38, Amer. Math. Soc., Providence, 1978.

3. C. Conley and R. Easton, Isolated invariant sets and isolating blocks, Trans. Amer. Math. Soc. 158 (1971), 35-61.

Institute of Mathematics, Academia Sinica, 100080 Beijing, China

E-mail address: ysx@math03.math.ac.cn 